УДК541.64:539.2

ISSN 1606-867X

DOI: https://doi.org/10.17308/kcmf.2019.21/2367

Поступила в редакцию 31.10 .2019

Подписана в печать 15.11.2019

\title{
Особенности электронной структуры и химических связей в композитах на основе полианилина, полученных бескислотным синтезом
}

по материалам XXIII Всероссийской конференции с международным участием «Рентгеновские и электронные спектры и химическая связь»

(Воронеж, 1-4 октября 2019)

\section{(c) 2019 В. А. Шматко ${ }^{\circledR}$, Т. Н. Мясоедова ${ }^{2}$, Т. А. Михайлова ${ }^{1}$, Г. Э. Яловега ${ }^{1}$}

${ }^{1}$ Южный федеральный университет

ул. Зорге, 5, 344090 Ростов-на-Дону, Российская Федерация

${ }^{2}$ Институт нанотехнологий, электроники и приборостроения ЮФу

ул. Шевченко, 2, корпус E, 347928 Таганрог, Ростовская область, Российская Федерация

\begin{abstract}
Аннотация. Композиты на основе полианилина и $\mathrm{CuCl}_{2} \cdot 2 \mathrm{H}_{2} \mathrm{O} / \mathrm{ZrOCl}_{2} \cdot 8 \mathrm{H}_{2} \mathrm{O}$ в качестве модифицирующих добавок получены методом химической полимеризации без добавления кислоты. Особенности электронной структуры и химических связей образцов исследованы методами ИК спектроскопии и спектроскопии рентгеновского поглощения. Микроструктура поверхности композитов исследовалась методом сканирующей электронной микроскопии. Полианилин в состав композитов входит в частично окисленной форме, степень окисления полимера зависит от типа модифицирующей добавки. Добавление $\mathrm{CuCl}_{2} \cdot 2 \mathrm{H}_{2} \mathrm{O} / \mathrm{ZrOCl}_{2} \cdot 8 \mathrm{H}_{2} \mathrm{O}$ в процессе синтеза увеличивает электропроводность образцов.
\end{abstract}

Ключевые слова: полианилин, композиты на основе полианилина, бескислотный синтез, рентгеновская спектроскопия поглощения, ИК спектроскопия.

\section{ВВЕДЕНИЕ}

Интерес к полианилину (ПАНИ, англ. РАNI), представителю класса электропроводящих полимеров, обусловлен возможностью получения на его основе материалов с самыми разнообразными физико-химическими свойствами $[1,2]$. Простота получения, низкая себестоимость, не токсичность, механическая прочность, термическая и химическая стабильность полианилина и композитов на его основе в совокупности со способностью варьирования свойств открывает широкие возможности для применения этих материалов в микроэлектронике, производства электрохромных дисплеев, датчиков, суперконденсаторов и прочего [3-6].

Полианилин представляет собой полимерную цепь повторяющихся звеньев, каждое из которых состоит из трех бензольных колец, разделённых аминными группами, и хиноидного кольца, окруженного иминными группами. В

Шматко Валентина Анатольевна, e-mail: shmatko86@mail.ru зависимости от соотношения количества бензоидных и хиноидных фрагментов полианилин может находиться в различных степенях окисления, которые связаны обратимыми переходами. Общая форма цепи полианилина представлена на рис. 1.

Свойства полианилина во многом зависят от его состояния окисления и протонирования. Наибольший интерес представляет полуокисленный полианилин, когда полимерная цепочка включает чередующиеся хиноидные и бензоидные структуры. Для улучшения функциональных<smiles>CN=C1C=CC(=Nc2ccc(Nc3ccc(Nc4ccc(C)cc4)cc3)cc2)C=C1</smiles>

Рис. 1. Структурная формула цепи полианилина

[Fig. 1. Schematic representation of polyaniline structure]

Контент доступен под лицензией Creative Commons Attribution 4.0 License.

The content is available under Creative Commons Attribution 4.0 License. 
свойств, например, проводимости, полианилин допируют протонными кислотами или создают композитные материалы на основе полианилина и неорганических материалов, таких как соли металлов [7, 8].

Основными методами получения полианилина и композитов на его основе являются электрохимические и химические методы. Недостатком электрохимического синтеза является малое количество синтезируемого материала, которое определяется размерами электродов. Методы химического синтеза полианилина являются более универсальными. Основные преимущества химического синтеза - простота и возможность получения высокого выхода полимера. Синтез полианилина и композитов на его основе обычно проводят в кислой среде при низких температурах. Основными недостатками при этом является использование коррозионностойких реакторов, необходимость многократного промывания продуктов реакции для удаления остатков кислоты, часто возникающая необходимость выведения примесей из полимера или его нейтрализации аммиаком.

Альтернативным способом получения полианилина на сегодняшний день является бескислотный синтез. Бескислотный синтез позволяет получать полианилин без использования специальных высококислотных реактивов и аммиака для нейтрализации полимера, что уменьшает трудоемкость данного способа и делает его более безопасным для окружающей среды [9-11]. Термическая обработка ПАНИ при различных температурах вызывает структурные изменения, такие как удаление легирующей примеси, окисление, разрыв цепи. Эти изменения в структуре влияют на свойства полимера в целом, и, в частности, на его проводимость, которая падает с увеличением температуры термической обработки [3, 12].

Поэтому большой интерес представляет изучение особенностей его химической и электронной структуры в зависимости от условий синтеза, как определяющей физико-химические свойства композита.

Целью работы являлся анализ электронной и химической структуры полианилина, синтезированного бескислотным методом с добавлением $\mathrm{CuCl}_{2} \cdot 2 \mathrm{H}_{2} \mathrm{O} / \mathrm{ZrOCl}_{2} \cdot 8 \mathrm{H}_{2} \mathrm{O}$ в качестве модифицирующих добавок и термостатированного при низкой температуре $\left(30^{\circ} \mathrm{C}\right)$, спектроскопическими методами (XANES, ИК) и изучение влияния модифицирующих добавок на электропроводность образующихся композитов.

\section{ЭКСПЕРИМЕНТАЛЬНЫЯ ЧАСТЬ}

Объекты и синтез. Для получения образцов чИстого ПАНИ и композитов ПАНИ/Ме $(\mathrm{Me}-\mathrm{Cu}$, $\mathrm{Zr}$ ) был использован метод химического окисления анилина без добавления кислоты. На первом этапе персульфат калия растворяли в дистиллированной воде. Затем добавляли анилин при постоянном перемешивании в течение 15 минут. На стадии полимеризации анилина вносили неорганические компоненты - раствор дигидрата хлорида меди $\mathrm{CuCl}_{2} \cdot 2 \mathrm{H}_{2} \mathrm{O}$ (образец ПАНИ-Сu) или оксихлорида циркония $\mathrm{ZrOCl}_{2} \cdot 8 \mathrm{H}_{2} \mathrm{O}$ (ПАНИ-Zr). Далее образцы термостатировали в течение 4 часов при температуре $30^{\circ} \mathrm{C}$. Соотношение компонент подбиралось таким образом, чтобы не препятствовать процессу полимеризации анилина. Это позволило совместить процессы полимеризации мономера и модифицирования.

Измерения ИК-спектров проводились на ИК-Фурье спектрометре ФМС 12-01. Измеряемые порошки были спрессованы в таблетки с добавлением $\mathrm{KBr}$ в соотношении 1:200.

Морфология композитных порошков ПАНИ/Me (Me - Cu, Zr) была исследована методом сканирующей электронной микроскопии на микроскопе LEO 1560 VP (ZEISS) в режиме регистрации вторичных электронов при высоком вакууме в рабочей камере ( $\mathrm{p} \sim 10^{-5}$ Torr) и ускоряющем напряжении $5 \mathrm{kV}$.

Экспериментальные спектры рентгеновского поглощения NEXAFS (Near edge X-ray absorption fine structure) K-края азота ПАНИ/Me (Me - Cu, Zr) получены в режиме регистрации выхода Ожеэлектронов при падении пучка излучения относительно поверхности образца $60^{\circ}$ на станции Materials science синхротронного центра Elettra (Триест, Италия). Давление остаточных газов в измерительной камере составляло $1.7 \cdot 10^{-9} \mathrm{mbar}$.

Спектры поглощения рентгеновского излучения XANES (X-ray absorption near edge structure) K-края меди измеряли на станции «Структурное материаловедение» Курчатовского источника синхротронного излучения (Москва, Россия). Регистрация рентгеновских спектров поглощения проводилась при комнатной температуре в режиме пропускания. Калибровка положения монохроматора проводилась по максимуму производной К-края поглощения фольги меди.

Электропроводность чистого полианилина и композитов ПАНИ-Сu, ПАНИ-Zr оценивалась по измеренным значениям удельного сопротивления. Для оценки величины сопротивления были спрессованы таблетки, на поверхность которых с помощью токопроводящего клея ЕРО-ТЕКН20Е 
с двух сторон прикреплялись медные пластинки. Сопротивление измерялось мультиметром KEYSIGHT 34410A.

\section{РЕЗУЛЬТАТЫ И ОБСУЖДЕНИЕ}

Известно, что морфология материала оказывает значительное влияние на свойства материала в целом $[13,14]$. На рис. 2 представлены микрофотографии композитов ПАНИ-Сu (рис. 2a) и ПАНИ-Zr (рис. 2b). Морфология обоих образцов сходна и представляет собой агломераты из пластинчатых (преимущественно) и палочкообразных структур, где пластинчатые структуры образованы металлами, а палочкообразные полианилином. Различия состоят в том, что в ПАНИ - Zr преобладает раздельная агломерация двух компонент (ПАНИ и Zr), в то время как в ПАНИ-Сu образующиеся агломера- ты представляют собой смесь из структур обоих компонент.

Каждая из форм полианилина (окисленные, восстановленные, допированные и пр.) имеет свои характерные особенности в ИК спектрах. Химические связи в композите были исследованы методом ИК-спектроскопии. На рис. 3 представлены ИК спектры пропускания комПозИтов ПАНИ-Cu, ПАНИ-Zr И чистоГо ПАНИ. Видно, что спектры ПАНИ и ПАНИ-Сu обладают практически одинаковым набором полос поглощения. Спектр образца ПАНИ-Zr отличается от этих спектров в области волновых чисел менее $1300 \mathrm{~cm}^{-1}$. Во всех спектрах присутствуют основные полосы при 1580 и $1504 \mathrm{~cm}^{-1}$, которые соответствуют колебаниям хинойдного $(\mathrm{N}=\mathrm{Q}=\mathrm{N})$ и бензольного $(\mathrm{N}-\mathrm{B}-\mathrm{N})$ колец соответственно [15]. Наличие обоих полос говорит о

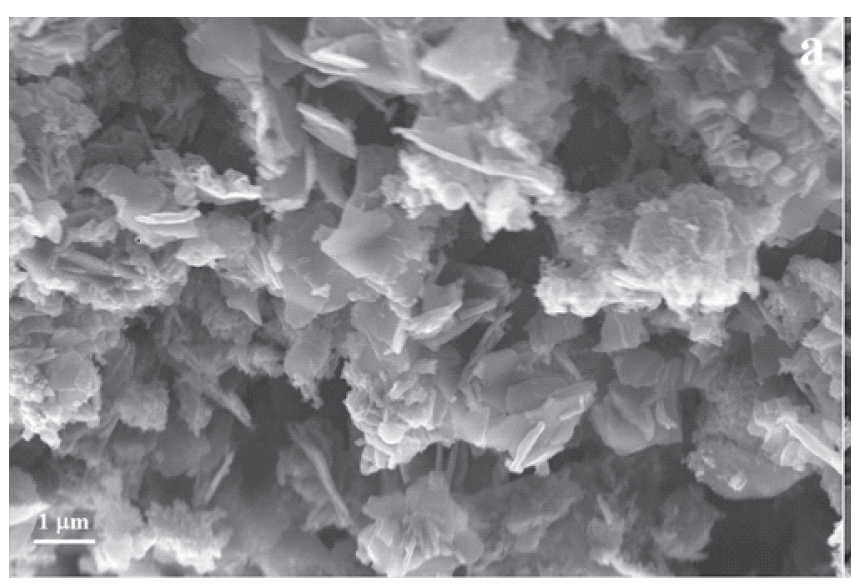

$a$

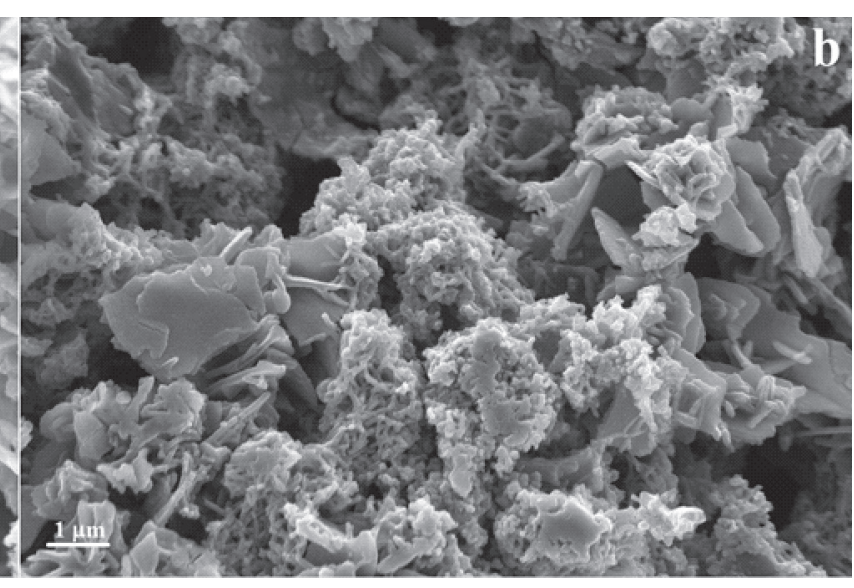

$b$

Рис. 2. МикрофотографиИ ПАНИ-Сu (a), ПАНИ-Zr (b)

[Fig. 2. SEM images of PANI-Cu $(a)$ and PANI-Zr $(b)$ ]

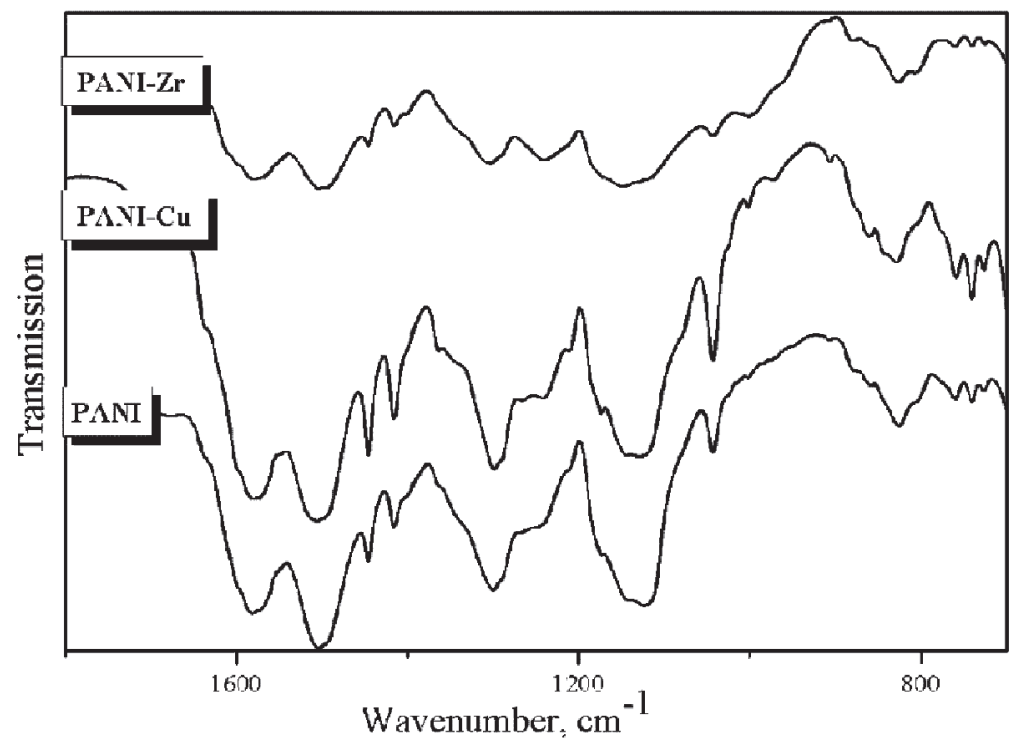

Рис. 3. ИК- спектры ПАНИ-Сu и ПАНИ-Zr в сравнении с чистым Полианилином (ПАНИ)

[Fig. 3. IR spectra of PANI-Cu, PANI-Zr and PANI] 
частично окисленном состоянии полианилина во всех образцах, так как для полностью окисленной формы характерно присутствие только хиноидных фрагментов, а для полностью восстановленной только бензоидных. Соотношение интенсивности этих полос варьируется в зависимости от образца, что указывает на различную степень окисления полианилина. Анализ соотношения интенсивностей показал, что степень окисления полианилина в ПАНИ-Zr больше чем в ПАНИ-Сu и близка к 0.5, что соответствует эмералдиновой форме.

Полоса при $1299 \mathrm{~cm}^{-1}$ относится к колебаниям связи C-N. Для образца ПАНИ-Zr эта полоса сдвигается на $7 \mathrm{~cm}^{-1}$ в сторону больших значений волновых чисел. Полоса поглощения около $1240 \mathrm{~cm}^{-1}$ связана с колебаниями связи $\mathrm{C}-\mathrm{N}^{+}$ [15]. Полоса поглощения при $1128 \mathrm{~cm}^{-1}$ в спектре ПАНИ, по-видимому, связана с колебаниями фрагмента B-NH ${ }^{+}-\mathrm{Q}$ [16]. При переходе к композитам происходит сдвиг полосы в сторону мень-

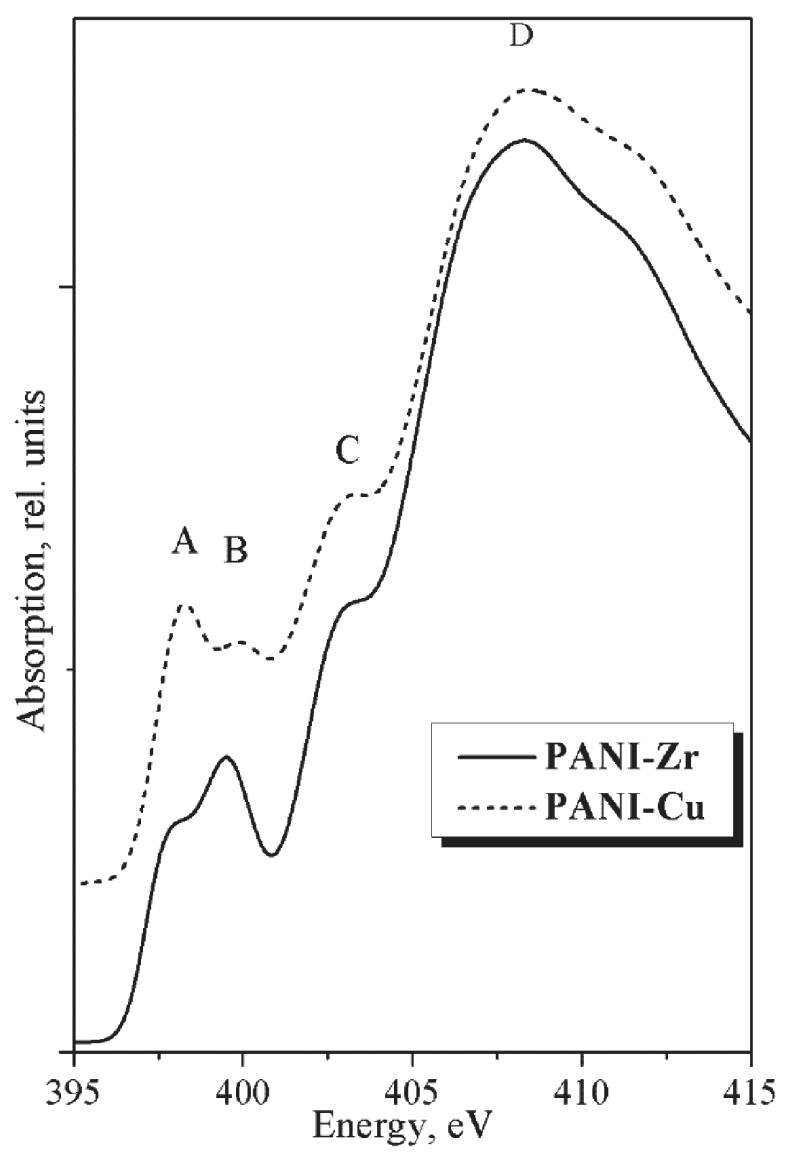

Рис. 4. Экспериментальные спектры рентгеновского поглощения NEXAFS вблизи главного К-края азота ПАНИ-Сu (пунктирная линия) и ПАНИ -Zr (сплошная линия)

[Fig. 4. N K edge NEXAFS spectra of PANI-Cu (dashed line), PANI-Zr(solid line)] ших значений волнового числа для ПАНИ-Сu и в сторону больших значений волнового числа для ПАНИ-Zr. Полоса поглощения при $830 \mathrm{~cm}^{-1}$ соответствует колебаниям С-Н связи. Приведенные спектральные данные указывают на то, что полимеризация анилина в присутствии $\mathrm{CuCl}_{2} \cdot 2 \mathrm{H}_{2} \mathrm{O}$ или $\mathrm{ZrOCl}_{2} \cdot 8 \mathrm{H}_{2} \mathrm{O}$ с дальнейшим термостатированием при $30^{\circ} \mathrm{C}$ приводит к образованию частично окисленной формы полианилина в композитах ПАНИ-Сu и ПАНИ-Zr. Причем степень окисления полианилина в ПАНИ-Zr больше, чем в ПАНИ-Cu. Сдвиг полос поглощения, соответствующих азотным связям, свидетельствует о взаимодействии катионов металлов с атомами азота полимерной цепи [7, 17].

Химическое состояние азота в полианилине определяет степень окисления и электронную структуру полимерной цепи. Исследования химического состояния азота в композитах в зависимости от типа модифицирующей добавки были проведены на основе анализа тонкой структуры спектров рентгеновского поглощения вблизи К-края азота. На рис. 4 представлено сопоставление спектров рентгеновского поглощения К-краев азота ПАНИ-Сu и ПАНИ-Zr. Toнкая структура спектров формируется в результате разрешенных дипольными правилами отбора переходов $1 s$ электронов атомов азота в свободные состояния зоны проводимости $2 p$. Спектры совпадают в области широкого максимума $D$ при энергии $~ 408 \mathrm{eV}$, который связан с делокализованными электронными $\sigma^{*}$ состояниями [18], тогда как в низкоэнергетической области $\pi^{*}$ состояний $\left(\mathrm{N} 1 s \rightarrow 2 p^{*}\right.$ электронные переходы) в спектрах наблюдаются значительные различия.

В низкоэнергетической области спектров NEXAFS K-краев азота наблюдается формирование трех спектральных особенностей (пики A, B, C), которые относятся к $=\mathrm{N}-,=\mathrm{NH}^{+}-$и $-\mathrm{NH}-$ $[19,20]$. Присутствие в спектре особенностей, относящихся не только к иминным и аминным группам, говорит о том, что полианилин вне зависимости от неорганического компонента находится в частично окисленном состоянии. Наблюдается перераспределение интенсивностей особенностей А и В, относящихся к различным азотогруппам, в зависимости от допирующего элемента. Количество иминных групп в образце ПАНИ-Zr в сравнении с ПАНИ-Cu уменьшается с одновременным ростом содержания аминных и положительно заряженных иминных групп. Этот результат подтверждает выводы, полученные на основе анализа данных ИК-спектроско- 
пии, о различной степени окисления и форме полианилина в зависимости от модифицирующей добавки.

Как известно, XANES спектры очень чувствительны к валентному состоянию и координации окружения поглощающего атома [21]. На рис. $5 a$ представлены спектры поглощения композита ПАНИ-Сu и спектры стандартных соединений меди $\left(\mathrm{CuCl}_{2} \cdot 2 \mathrm{H}_{2} \mathrm{O}, \mathrm{CuO}, \mathrm{Cu}_{2} \mathrm{O}\right)$. Отличие спектра композита от спектров стандартов говорит о том, что локальная структура меди в ПАНИ-Сu не совпадает с локальной структурой меди в исходной компоненте - дигидрохлориде меди, в оксидах $\mathrm{CuO}, \mathrm{Cu}_{2} \mathrm{O}$. Форма спектра ПАНИ-Сu, энергетическое положение края поглощения $(8984.5 \mathrm{eV})$, который определялся по первому максимуму производной, и «белой линии» $(8997.3 \mathrm{eV})$, наличие предкраевых особенностей (пик А и плечо В) говорит о том, что ионы меди в ПАНИ-Сu находятся в окисленной форме $2^{+}$. Энергетическое положение и интенсивность предкраевых пиков А и В является индикатором количества и расположения лигандов атомов меди. Плечо В со стороны низких энер-

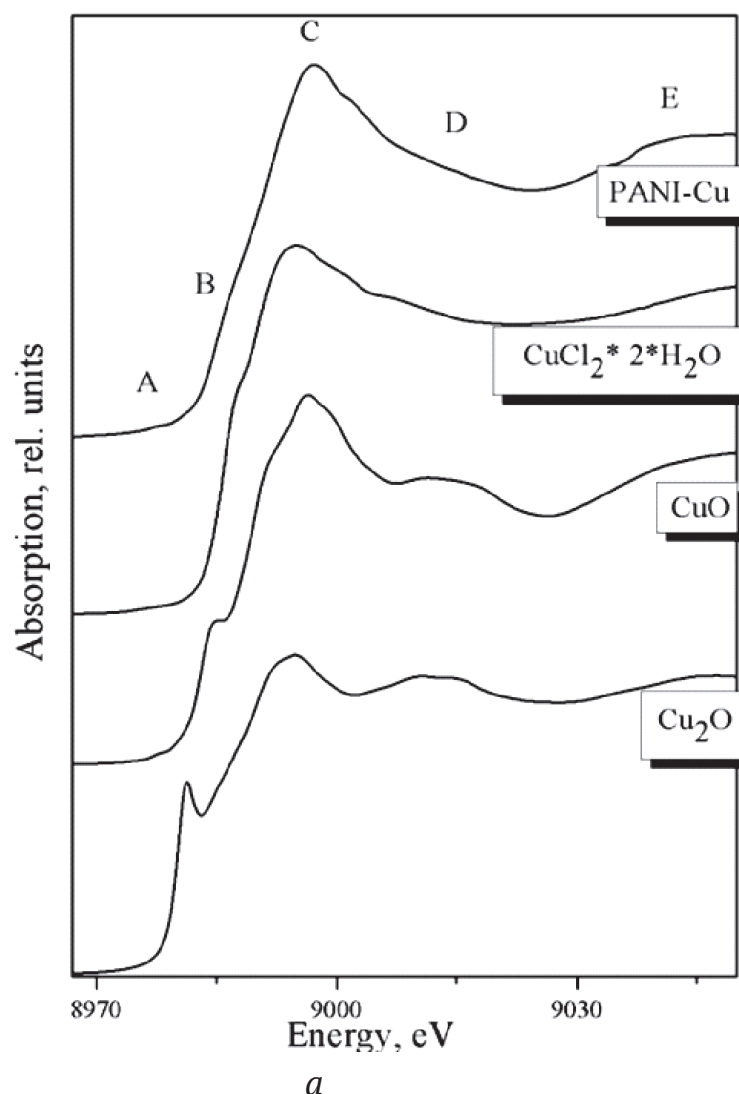

гий главного максимума поглощения связано с переносом заряда лиганд-металл и указывает на наличие атомов в аксиальных позициях и 6кратную координацию иона меди [22]. Наличие слабоинтенсивного пика А также подтверждает 6-кратную координацию иона меди. Отличия спектра композита от спектров стандартов связаны с тем, что в композите присутствуют ионы меди с различной локальной структурой. Вероятно, что ионы меди внутри пластинчатой медьсодержащей микроструктуры имеют локальное окружение как в $\mathrm{CuCl}_{2} \cdot 2 \mathrm{H}_{2} \mathrm{O}$, в то время как локальное окружение ионов меди на поверхности этой микроструктуры отличается из-за соседствующих микроструктур полианилина. В этом случае в ближайшее окружение меди могут входить атомы азота полианилина [23, 24].

На рис. $5 b$ представлено сопоставление экспериментальных спектров XANES для ПАНИ-Zr и стандартных соединений $-\mathrm{ZrO}_{2}, \mathrm{ZrOCl}_{2} \cdot 8 \mathrm{H}_{2} \mathrm{O}$. По положению «белой линии» и края поглощения спектр композита совпадает со спектром $\mathrm{ZrOCl}_{2} \cdot 8 \mathrm{H}_{2} \mathrm{O}$, следовательно, цирконий в композите находится в окисленной форме $4^{+}$[25].

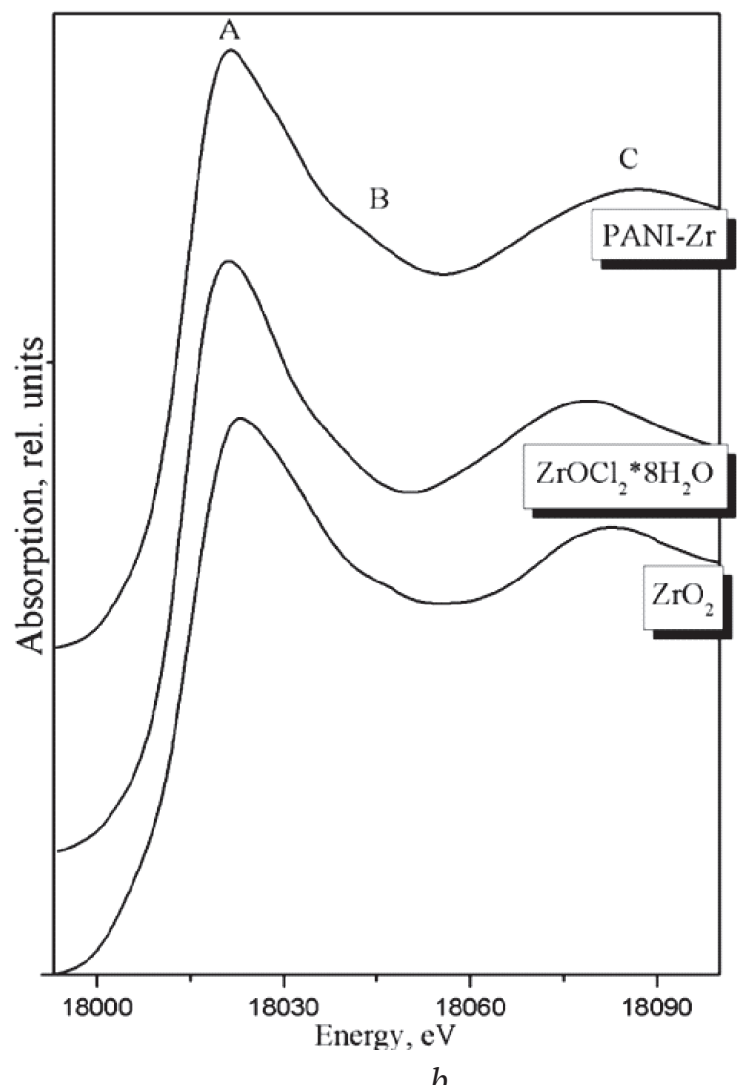

Рис. 5. Экспериментальные спектры рентгеновского поглощения XANES вблизи главного К-края меди для ПАНИ-Сu $(a)$ и главного К-края циркония для ПАНИ-Zr в сопоставлении со спектрами стандартов ПАНИ-Zr, $\mathrm{ZrO}_{2}, \mathrm{ZrOCl}_{2} \cdot 8 \mathrm{H}_{2} \mathrm{O}(b)$

[Fig. 5. $\mathrm{Cu} \mathrm{K}$ edge XANES spectra of PANI-Cu, $\mathrm{CuCl}_{2} \cdot 2 \mathrm{H}_{2} \mathrm{O}, \mathrm{CuO}, \mathrm{Cu}_{2} \mathrm{O}(a)$ and $\mathrm{Zr} \mathrm{K}$ edge XANES spectra of PANI-Zr, $\left.\mathrm{ZrO}_{2}, \mathrm{ZrOCl}_{2} \cdot 8 \mathrm{H}_{2} \mathrm{O}(b)\right]$ 
Однако полного согласия не наблюдается, что является следствием того, что локальная структура циркония изменилась при формировании композита. Вероятно, изменения в локальной структуре ионов циркония также связано с влиянием полимерной цепи. Смещение плеча В и пика С в область высоких энергий указывает на уменьшение межатомных расстояний циркония и ближайших соседей в сравнении с расстояниями $\mathrm{Zr}-\mathrm{O}$ в $\mathrm{ZrOCl}_{2} \cdot 8 \mathrm{H}_{2} \mathrm{O}$.Спектр композита также не совпадает со спектром оксида циркония $\mathrm{ZrO}_{2}$, поэтому можно заключить, что локальная структура ионов циркония в композите и оксиде не совпадают.

Измерение удельного сопротивления образцов ПАНИ, ПАНИ-Сu, ПАНИ-Zr показали, что введение солей металлов в процессе синтеза позволяет повысить электропроводность ком-

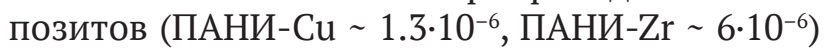
по сравнению с чистым ПАНИ (удельная проводимость $\sim 7 \cdot 10^{-7}$ ) на порядок. Некоторое увеличение электропроводности ПАНИ- $\mathrm{Zr}$ по сравнению с ПАНИ-Сu может быть связано с большей степенью окисления и с большим содержанием протонированных иминных групп в образце ПАНИ-Zr.

\section{ЗАКЛЮЧЕНИЕ}

Бескислотный синтез методом химической полимеризации анилина в присутствии $\mathrm{CuCl}_{2} \cdot 2 \mathrm{H}_{2} \mathrm{O} / \mathrm{ZrOCl}_{2} \cdot 8 \mathrm{H}_{2} \mathrm{O}$ позволил получить композитные материалы различной морфологии с содержанием частично окисленной формы полианилина. Установлено, что степень окисления полианилина в ПАНИ-Zr выше, чем в ПАНИ-Cu. Исследования химических связей и электронной структуры показали, что для образца, полученного с добавлением оксихлорида циркония, характерно большее содержание протонированных азотогрупп, чем для образца, полученного с добавлением гидрохлорида меди. Модифицирование полианилина солями переходных металлов (Zr, Cu) повышает электропроводность композитов по сравнению с чистым полианилином.

\section{ИСТОЧНИК ФИНАНСИРОВАНИЯ}

Работа выполнена при финансовой поддержке Гранта Президента МК-4933.2018.2.

\section{БЛАГОДАРНОСТИ}

Авторы выражают благодарность синхротронному центру Elettra (станция Material Science) и сотрудникам станции «Структурное матери- аловедение» НИЦ «Курчатовский институт» за предоставление времени, а также М. М. Бржезинской за проведение СЭМ измерений.

\section{КОНФЛИКТ ИНТЕРЕСОВ}

Авторы декларируют отсутствие явных и потенциальных конфликтов интересов, связанных с публикацией настоящей статьи

\section{СПИСОК ЛИТЕРАТУРЫ}

1. Ćirić-Marjanović G. Recent advances in polyaniline research: Polymerization mechanisms, structural aspects, properties and applications // Synthetic Metals, 2013, v. 177, pp.1-47.DOI: https://doi. org/10.1016/j.synthmet.2013.06.004

2. Боева Ж. А., Сергеев В.Г. Полианилин: синтез, свойства и применение // Высокомолекулярные соединения. Серия C, 2014, т. 56(1), с. 153-164. DOI: https://doi.org/10.7868/S2308114714010038

3. Benabdellah A., Ilikti H., Belarbi H., Fettouhi B., Ait Amer A., Hatti M. Effects of the synthesis temperature on electrical properties of polyaniline and their electrochemical characteristics onto silver cavity microelectrode Ag/C-EM // Int. J. Electrochem. Sci., 2011, v. 6, pp. $1747-1759$.

4. Kelly F. M., Meunier L., Cochrane C., Koncar V. Polyaniline application as solid state electrochromic in a flexible textile display // Displays, 2013, v. 34 (1), pp. 1-7. DOI: https://doi.org/10.1016/j.displa.2012.10.001

5. Lobotka P., Kunzo P., Kovacova E., Vavra I., Krizanova Z., Smatko V., Stejskal J., Konyushenko E. N., Omastova M., Spitalsky Z., Micusik M., Krup I. Thin polyaniline and polyaniline/carbon nanocomposite films for gas sensing // Thin Solid Films, v. $519(12,1)$, pp. 4123-4127. DOI: https://doi.org/10.1016/j. tsf.2011.01.177

6. Wang H., Linc J., Shen Z.X. Polyaniline (PANi) based electrode materials for energy storage and conversion // Journal of Science: Advanced Materials and Devices, 2016, v. 1 (3), pp. 225-255. DOI: https://doi. org/10.1016/j.jsamd.2016.08.001

7. Иванова Н. М., Соболева Е. А., Висурханова Я. А., Кирилюс И. В. Электрокаталитическая активность полианилин-медных композитов в электрогидрировании р-нитроанилина // Электрохимия, 2015, т. 51 (2), с. 197-204. DOI: https://doi. org/10.7868/S042485701502005X

8. Матнишян А.А., Ахназарян Т. Л., Абагян Г. В., Бадалян Г. Р., Петросян С. И., Кравцова В. Д. Синтез и исследование нанокомпозитов полианилина с окислами металлов // ФТТ, 2011, т. 53 (8), с. 16401644. DOI: https://doi.org/10.1134/ S1063783411080178

9. Zhu Y., He H., Wan M., Jiang L. Rose-like microstructures of polyaniline by using a simplified tem- 
plate-free method under a high relative humidity // Macromol. Rapid Commun., 2008, v. 29 (21), pp. 17051710. DOI: https://doi.org/10.1002/marc.200800294

10. Konyushenko E.N., Stejskal J., Šeděnková I., Trchová M., Sapurina I., Cieslar M., Prokeš J. Polyaniline nanotubes: conditions of formation // Polym. Int, 2006, v. 55, pp. 31-39. DOI: https://doi.org/10.1002/ pi.1899

11. Trchová M., Šeděnková I., Konyushenko E. N., Stejskal J., Holler P., Ćirić-Marjanović G. Evolution of polyaniline nanotubes: The oxidation of aniline in water // J. Phys. Chem. B, 2006, v. 110(19), pp. 94619468. DOI: https://doi.org/10.1021/jp057528g

12. Bhadra S., Khastgir D. Extrinsic and intrinsic structural change during heat treatment of polyaniline // Polymer Degradation and Stability, 2008, v. 93 (6), pp. 1094-1099. DOI: https://doi.org/10.1016/j. polymdegradstab.2008.03.013

13. Yalovega G. E., Myasoedova T. N., Shmatko V. A., Brzhezinskaya M. M., Popov Y. V. Influence of $\mathrm{Cu} / \mathrm{Sn}$ mixture on the shape and structure of crystallites in copper-containing films: Morphological and X-ray spectroscopy studies // Applied Surface Science, 2016, v. 372, pp. 93-99. DOI: https://doi.org/10.1016/ j.apsusc.2016.02.245

14. Domashevskaya E. P., Hadia N. M. A., Ryabtsev S. V., Seredin P. V. Structure and photoluminescence properties of $\mathrm{SnO}_{2}$ nanowires synthesized from SnO powder // Kondensirovannye sredy i mezhfaznye granitsy [Condensed Matter and Interphases], 2009, v. 11(1), C. 5-9

15. Baibarac M., Baltog I., Lefrant S., Mevellec J. Y., Chauvet O. Polyaniline and carbon nanotubes based composites containing whole units and fragments of nanotubes // Chem. Mater., 2003, v. 15, pp. 4149-4156. DOI: https://doi.org/10.1021/cm021287x

16. Окотруб А. В., Асанов И. П., Галкин П. С., Булушева Л. Г., Чехова Г. Н., Куреня А. Г., Шубин Ю. В. Композиты на основе полианилина и ориентированных углеродных нанотрубок // Высокомолекулярные соединения Серия Б, 2010, т. 52 (2), с. 351359.

17. Wang S., Tan Z., Li Y., Suna L., Zhang T. Synthesis, characterization and thermal analysis of polyaniline/ $\mathrm{ZrO}_{2}$ composites // Thermochimica Acta, 2006, v.441,pp. 191-194.DOI: https://doi.org/10.1016/ j.tca.2005.05.020

18. Ullah R., Bowmaker G.A., Laslau C., Waterhouse G. I. N., Zujovic Z. D., Ali K., Shah A.-U.-H. A.,
Travas-Sejdic J. Synthesis of polyaniline by using $\mathrm{CuCl}_{2}$ as oxidizing agent // Synthetic Metals, 2014, v. 198, pp. 203-211. DOI: https://doi.org/10.1016/j.synthmet.2014.10.005

19. Izumi C. M., Constantino V. R., Temperini M. L. Spectroscopic characterization of polyaniline formed by using copper(II) in homogeneous and MCM-41 molecular sieve media // J. Phys. Chem. B, 2005, v. 109, pp. 22131-22140. DOI: https://doi.org/10.1021/ jp051630w

20. Magnuson M., Guo J.-H., Butorin S.M., Agui A., Sethe C., Nordgren J. The electronic structure of polyaniline and doped phases studied by soft x-ray absorption and emission spectroscopies // J. Chem. Phys., 1999, v. 111, pp. 4756-4761. DOI: https://doi.org/10. 1063/1.479238

21. Домашевская Э. П., Сторожилов С.А., Турищев С. Ю., Кашкаров В. М., Терехов В. А., Стогней О. В., Калинин Ю. Е., Ситников А. В., Молодцов С. Л. XANES- И USXES-исследования межатомных взаимодействий в нанокомпозитах $\left(\mathrm{Co}_{41} \mathrm{Fe}_{39} \mathrm{~B}_{20}\right) \mathrm{x}\left(\mathrm{SiO}_{2}\right)_{1-x} / / \Phi T T, 2008$, т. 50 (1), c. 135141.

22. Gaur A., Klysubun W., Sonic B., Shrivastav D., Prasad J., Srivastava K. Identification of different coordination geometries by XAFS in copper(II) complexes with trimesic acid // Journal of Molecular Structure, 2016, v. 1121, pp. 119-127. DOI: https://doi. org/10.1016/j.molstruc.2016.05.066

23. Fulton J. L., Hoffmann M. M., Darab J. G., Palmer B. J. Copper(I) and copper(II) coordination structure under hydrothermal conditions at $325^{\circ} \mathrm{C}$ : an X-ray absorption fine structure and molecular dynamics study // J. Phys. Chem. A., 2000, v. 104, pp. 11651-11663. DOI: https://doi.org/10.1021/ jp001949a

24. Porto A. O., Pernaut J. M., Daniel H., Schilling P. J., Martins M. C. Alves X-ray absorption spectroscopy of iron-doped conducting polymers // Synthetic Metals, 1999, v. 104, pp. 89-94. DOI: https://doi. org/10.1016/S0379-6779(99)00025-9

25. Zhang Y., Addison O., Gostin P. F., Morrell A., Cook A. J. M. C., Liens A., Wu J., Ignatyev K., Stoica M., Davenport A. In-situ synchrotron X-ray characterization of corrosion products in $\mathrm{Zr}$ artificial pits in simulated physiological solutions // J. Electrochem. Soc, 2017, v. 164(14), pp. 1003-1012. DOI: https://doi. org/10.1149/2.0671714jes 
Received 31.10.2019

Accepted 15.11.2019

\title{
Features of the Electronic Structure and Chemical Bonds ofPolyaniline-Based Composites Obtained by Acid-Free Synthesis
}

\author{
(c) 2019 V. A. Shmatko ${ }^{\otimes 1}$, T. N. Mysoedova ${ }^{2}$, T. A. Mikhailova ${ }^{1}$, G. E. Yalovega ${ }^{1}$ \\ ${ }^{1}$ Southern Federal University \\ 5, Zorge str., 344090 Rostov-on-Don, Russian Federation \\ ${ }^{2}$ Southern Federal University, Institute of Nanotechnologies, Electronics and Equipment Engineering \\ 2, Chekhov str., 347928 Taganrog, Russian Federation
}

\begin{abstract}
Purpose. The analysis of the electronic and chemical structure of polyaniline, synthesized by the acid-free method with the addition of $\mathrm{CuCl}_{2} \cdot 2 \mathrm{H}_{2} \mathrm{O} / \mathrm{ZrOCl}_{2} \cdot 8 \mathrm{H}_{2} \mathrm{O}$ modifying agent and thermostated at low temperature $\left(30^{\circ} \mathrm{C}\right)$, by spectroscopic methods (XANES, IR). The investigation of the effect of modifying agents on the electrical conductivity of composites.

Methods. PANI/Me composites ( $\mathrm{Me}-\mathrm{Cu}, \mathrm{Zr}$ ) were obtained by acid-free chemical oxidation of aniline.Potassium persulfate was dissolved in distilled water and then aniline was added with constant stirring for 15 minutes. Solutions of $\mathrm{CuCl}_{2} \cdot 2 \mathrm{H}_{2} \mathrm{O} / \mathrm{ZrOCl}_{2} \cdot 8 \mathrm{H}_{2} \mathrm{O}$ were added as a modifying agent. Then samples were thermostated at $30^{\circ} \mathrm{C}$ for 4 hours.A surface morphological study of the samples was carried out using a scanning electron microscopy with an accelerating voltage of $5 \mathrm{kV}$. The electronic and chemical structure of PANI/Me composites was investigated by IR spectroscopy and $\mathrm{X}$-ray absorption spectroscopy.

Results. The addition of $\mathrm{CuCl}_{2} \cdot 2 \mathrm{H}_{2} \mathrm{O} / \mathrm{ZrOCl}_{2} \cdot 8 \mathrm{H}_{2} \mathrm{O}$ during the chemical polymerization of aniline by the acid-free method leads to the formation of partially oxidized polyaniline. The oxidation state of PANI depends on the type of modifying agent. The oxidation state of polyaniline in PANI-Zr is higher than in PANI-Cu. The morphology of both samples was similar and represented by agglomerates of lamellar (mainly) and rod-like structures. However, in PANI-Cu agglomerates of mixed type were revealed, while in PANI-Zr agglomerates consisted of one type of microstructures. The addition of metal-containing components improved the conductivity of the samples. PANI-Zr contained more protonated nitrogen groups compared to PANI- $\mathrm{Cu}$, which improved its conductivity.

Conclusion. Acid-free synthesis by the chemical polymerization of aniline method in the presence of $\mathrm{CuCl}_{2} \cdot 2 \mathrm{H}_{2} \mathrm{O} / \mathrm{ZrOCl}_{2} \cdot 8 \mathrm{H}_{2} \mathrm{O}$ made it possible to obtain composite materials of various morphologies with partially oxidized form of polyaniline. It was found that the oxidation state of polyaniline in PANI-Zr is higher than in PANI-Cu. Studies of chemical and electronic structure showed that the sample obtained with the addition of zirconium oxychloride is characterized by a higher content of protonated nitrogen groups than the sample obtained with the addition of copper hydrochloride. Modification of polyaniline with transition metal salts $(\mathrm{Zr}, \mathrm{Cu})$ improved the electrical conductivity of composites when compared with pure polyaniline.
\end{abstract}

Keywords: polyaniline, composites based on polyaniline, acid-free synthesis, X-ray absorption spectroscopy, IR spectroscopy.

\section{SOURCE OF FINANCING}

The study was supported by the grant of the President of Russian Federation for Young Scientists (Grant MK-4933.2018.2)."

\section{AKNOWLEDGMENTS}

The authors are grateful to theElettra synchrotron centre (Material Science station), employees of the

Valentina A.Shmatko, e-mail:shmatko86@mail.ru
National Research Centre "Kurchatov Institute" (Structural materials science station), and M. M. Brzhezinskaya for SEM measurements.

\section{REFERENCE}

1. Ćirić-Marjanović G. Recent advances in polyaniline research: Polymerization mechanisms, structural aspects, properties and applications. Synthetic Metals, 2013, v. 177, pp. 1-47. DOI: https://doi. org/10.1016/j.synthmet.2013.06.004 
2. Boeva Zh. A., Sergeyev V. G. Polyaniline: Synthesis, properties, and application. Polymer Science. Series C., 2014, v. 56(1), pp. 144-153. DOI: https://doi. org/10.1134/S1811238214010032

3. Benabdellah A., Ilikti H., Belarbi H., Fettouhi B., Ait Amer A., Hatti M. Effects of the synthesis temperature on electrical properties of polyaniline and their electrochemical characteristics onto silver cavity microelectrode Ag/C-EM. Int. J. Electrochem. Sci., 2011, v. 6, pp. 1747 - 1759 .

4. Kelly F. M., Meunier L., Cochrane C., Koncar V. Polyaniline application as solid state electrochromic in a flexible textile display. Displays, 2013, v. 34 (1), pp.1-7. DOI: https://doi.org/10.1016/j.displa.2012.10.001

5. Lobotka P., Kunzo P., Kovacova E., Vavra I., Krizanova Z., Smatko V., Stejskal J., Konyushenko E. N., Omastova M., Spitalsky Z., Micusik M., Krup I. Thin polyaniline and polyaniline/carbon nanocomposite films for gas sensing. Thin Solid Films, v. $519(12,1)$, pp. 4123-4127. DOI: https://doi.org/10.1016/j. tsf.2011.01.177

6. Wang H., Linc J., Shen Z.X. Polyaniline (PANi) based electrode materials for energy storage and conversion. Journal of Science: Advanced Materials and Devices, 2016, v. 1 (3), pp. 225-255. DOI: https://doi. org/10.1016/j.jsamd.2016.08.001

7. Ivanova N. M., Soboleva E. A., Visurkhanova Y. A., Kirilyus I. V. Electrocatalytic activity of polyanilinecopper composites in electrohydrogenation of p-nitroaniline. Russian Journal of Electrochemistry, 2015, v. 51(2), pp. 166-173. DOI: https://doi.org/10.1134/ S1023193515020056

8. Matnishyan A. A., Akhnazaryan T. L., Abagyan G. V., Badalyan G. R., Petrosyan S. I., Kravtsova V. D. Synthesis and study of polyaniline nanocomposites with metal oxides. Physics of the Solid State, 2011, v. 53 (8), c. 1727-1731. DOI: https://doi. org/10.1134/s1063783411080178

9. Zhu Y., He H., Wan M., Jiang L. Rose-like microstructures of polyaniline by using a simplified template-free method under a high relative humidity. Macromol. Rapid Commun., 2008, v. 29 (21), pp. 17051710. DOI: https://doi.org/10.1002/marc.200800294

10. Konyushenko E.N., Stejskal J., Šeděnková I., Trchová M., Sapurina I., Cieslar M., Prokeš J. Polyaniline nanotubes: conditions of formation. Polym. Int, 2006, v. 55, pp. 31-39. DOI: https://doi.org/10.1002/ pi. 1899

11. Trchová M., Šeděnková I., Konyushenko E.N., Stejskal J., Holler P., Ćirić-Marjanović G. Evolution of polyaniline nanotubes: The oxidation of aniline in water. J. Phys. Chem. B, 2006, v. 110(19), pp. 9461-9468. DOI: https://doi.org/10.1021/jp057528g

12. Bhadra S., Khastgir D. Extrinsic and intrinsic structural change during heat treatment of polyaniline. Polymer Degradation and Stability, 2008, v. 93 (6), pp. 1094-1099. DOI: https://doi.org/10.1016/j.polymdegradstab.2008.03.013

13. Yalovega G. E., Myasoedova T. N., Shmatko V. A., Brzhezinskaya M. M., Popov Y. V. Influence of $\mathrm{Cu} / \mathrm{Sn}$ mixture on the shape and structure of crystallites in copper-containing films: Morphological and $\mathrm{X}$-ray spectroscopy studies. Applied Surface Science, 2016, v. 372, pp. 93-99. DOI: https://doi.org/10.1016/ j.apsusc.2016.02.245

14. Domashevskaya E. P., Hadia N. M. A., Ryabtsev S. V., Seredin P. V. Structure and photoluminescence properties of $\mathrm{SnO}_{2}$ nanowires synthesized from SnO powder. Kondensirovannye sredy i mezhfaznye granitsy [Condensed Matter and Interphases], 2009, v. 11(1), p. 5-9. (in Russ.)

15. Baibarac M., Baltog I., Lefrant S., Mevellec J. Y., Chauvet O. Polyaniline and carbon nanotubes based composites containing whole units and fragments of nanotubes. Chem. Mater., 2003, v. 15, pp. 4149-4156. DOI: https://doi.org/10.1021/cm021287x

16. Okotrub A. V., Asanov I. P., Galkin P. S., Bulusheva L. G., Chehova G. N., Kurenja A. G., Shubin Ju. V. Composites based on polyaniline and aligned carbon nanotubes. Polymer Science - Series B, 2010, v. 52 (1-2), pp. 101-108.

17. Wang S., Tan Z., Li Y., Suna L., Zhang T. Synthesis, characterization and thermal analysis of polyaniline/ $/ \mathrm{ZrO}_{2}$ composites. Thermochimica Acta, 2006, v. 441, pp. 191-194. DOI: https://doi.org/10.1016/j. tca.2005.05.020

18. Ullah R., Bowmaker G. A., Laslau C., Waterhouse G. I. N., Zujovic Z. D., Ali K., Shah A.-U.-H. A., Travas-Sejdic J. Synthesis of polyaniline by using $\mathrm{CuCl}_{2}$ as oxidizing agent. Synthetic Metals, 2014, v. 198, pp. 203-211. DOI: https://doi.org/10.1016/j.synthmet.2014.10.005

19. Izumi C. M., Constantino V. R., Temperini M. L. Spectroscopic characterization of polyaniline formed by using copper(II) in homogeneous and MCM-41 molecular sieve media. J. Phys. Chem. B, 2005, v. 109, pp. 22131-22140. DOI: https://doi.org/10.1021/ jp051630w

20. Magnuson M., Guo J.-H., Butorin S. M., Agui A., Sethe C., Nordgren J. The electronic structure of polyaniline and doped phases studied by soft x-ray absorption and emission spectroscopies. J. Chem. Phys., 1999, v. 111, pp. 4756-4761. DOI: https://doi.org/10.1063/ 1.479238

21. Domashevskaya E. P, Storozhilov S. A. Turishchev S. Yu. Kashkarov V. M., Terekhov V. A., Stogney O. V., Kalinin Yu. E., Sitnikov A. V., Molodtsov S. L. XANES and USXES studies of interatomic interactions in $\left(\mathrm{Co}_{41} \mathrm{Fe}_{39} \mathrm{~B}_{20}\right) \mathrm{x}\left(\mathrm{SiO}_{2}\right)_{1-\mathrm{x}}$ nanocomposites. Physics of the Solid State, 2008, v. 50 (1), c. 139-145.

22. Gaur A., Klysubun W., Sonic B., Shrivastav D., Prasad J., Srivastava K. Identification of different coordination geometries by XAFS in copper(II) com- 
plexes with trimesic acid. Journal of Molecular Structure, 2016, v. 1121, pp. 119-127. DOI: https://doi.org/ 10.1016/j.molstruc.2016.05.066

23. Fulton J. L., Hoffmann M. M., Darab J. G., Palmer B. J. Copper(I) and copper(II) coordination structure under hydrothermal conditions at $325^{\circ} \mathrm{C}$ : an X-ray absorption fine structure and molecular dynamics study. J. Phys. Chem. A., 2000, v. 104, pp. 1165111663. DOI: https://doi.org/10.1021/jp001949a

24. Porto A. O., Pernaut J. M., Daniel H., Schilling P. J., Martins M. C. Alves X-ray absorption spec- troscopy of iron-doped conducting polymers. Synthetic Metals, 1999, v. 104, pp. 89-94. DOI: https://doi. org/10.1016/S0379-6779(99)00025-9

25. Zhang Y., Addison O., Gostin P. F., Morrell A., Cook A. J. M. C., Liens A., Wu J., Ignatyev K., Stoica M., Davenport A. In-situ synchrotron X-ray characterization of corrosion products in $\mathrm{Zr}$ artificial pits in simulated physiological solutions. J. Electrochem. Soc, 2017, v. 164(14), pp. 1003-1012. DOI: https://doi.org/ $10.1149 / 2.0671714$ jes
Шматко Валентина Анатольевна - к. ф -м. н, преподаватель кафедры физики наносистем и спектроскопии физического факультета ЮФУ, Ростов-на-Дону, Российская Федерация; e-mail: shmatko86@mail.ru. ORCID iD: https://orcid. org/0000-0002-7191-7970.

Мясоедова Татьяна Николаевна - к. ф-м. н. доцент кафедры техносферной безопасности и химии, Институт нанотехнологий, электроники и приборостроения ЮФУ, Таганрог, Российская Федерация; e-mail: ntn_79@mail.ru.

Михайлова Татьяна Андреевна - м. н. с. Междисциплинарной научной лаборатории аналитических технологий «Аналитикс» ЮФУ, Ростов-на-Дону, Российская Федерация; e-mail: tanymisha@mail.ru.

Яловега Галина Эдуардовна - д. ф.-м. н., профессор кафедры физики наносистем и спектроскопии физического факультета ЮФУ, Ростов-на-Дону, Российская Федерация; e-mail: yalovega1968@mail.ru. ORCID iD: https://orcid. org/0000-0002-0157-6955.
Valentina A. Shmatko - Cand. Sci. (Phys. and Math), Lecturer Department of Physics Nanosystem and Spectroscopy, Southern Federal University, Rostov-on-Don, Russia Federation; e-mail: shmatko86@mail.ru. ORCID iD: https://orcid.org/00000002-7191-7970.

Tatiana N. Myasoedova - Cand. Sci. (Phys. and Math), Associate Professor, Department of Technosphere Safety and Chemistry, Southern Federal University, Institute of Nanotechnologies, Electronics and Equipment Engineering, Taganrog, Russia Federation; e-mail:ntn_79@mail.ru.

Mikhailova Tatiana A. - Researcher, Laboratory "AnalytiX", Southern Federal University, Rostovon-Don, Russia Federation; e-mail: tanymisha@ mail.ru.

Galina E. Yalovega - Dr. Sci. (Phys. And Math), Professor, Department of Physics Nanosystem and Spectroscopy, Southern Federal University, Rostovon-Don, Russia Federation; e-mail: yalovega1968@ mail.ru. ORCID iD: https://orcid.org/0000-00020157-6955. 